\title{
Un modèle multi-agents pour étudier les politiques d'affectation des terres et leurs impacts sur les dynamiques pastorales et territoriales au Ferlo (Sénégal)
}

\author{
Alassane Bah ${ }^{1}$ \\ Ibra Touré ${ }^{2}$ \\ Christine Fourage ${ }^{3}$ \\ Ibrahima Diop Gaye ${ }^{4}$ \\ Grégoire Leclerc ${ }^{5}$ \\ Arame Soumaré ${ }^{2}$ \\ Alexandre Ickowicz ${ }^{7}$ \\ Amadou Tamsir Diop 8 \\ 1 École supérieure polytechnique \\ PPZS /UMI UMMISCO 209 \\ Département génie informatique \\ Ucad \\ BP 15915 Dakar-Fann \\ Sénégal \\ <alassane.bah@gmail.com> \\ ${ }^{2}$ Cirad \\ URP 68 PPZS, ISRA-LNERV \\ Route de Front de Terre \\ BP 2057 Dakar Hann \\ Senegal \\ <ibra.toure@cirad.fr> \\ ${ }^{3}$ Université catholique de l'Ouest \\ CERIPSA \\ 3, place André Leroy, BP 10808 \\ 49008 Angers cedex 01, France \\ 4 ENEA-PPZS ATEGU \\ Aménagement du Territoire, Environnement \\ ATEGU BP 5084, Dakar \\ Senegal \\ ${ }^{5}$ Cirad UR GREEN TA C-47 / F \\ Campus international de Baillarguet \\ 34398 Montpellier cedex 5 \\ France \\ <gregoire.leclerc@cirad.fr> \\ ${ }^{6}$ Ucad \\ Faculté des lettres et sciences humaines \\ Département de géographie \\ BP 5005, Dakar, Sénégal \\ ${ }^{7}$ Cirad \\ UMR 84 ERRC \\ Campus SupAgro-Inra \\ 2, place Pierre Viala \\ 34060 Montpellier cedex 1, France \\ <alexandre.ickowicz@cirad.fr> \\ 8 Isra \\ URP 68 PPZS \\ Isra-LNERV, Route du Front de Terre \\ BP 2057, Dakar Hann, Sénégal \\ <amtadiop@orange.sn>
}

Tirés à part : A. Bah

\begin{abstract}
Résumé
L'état actuel des paysages et des territoires sahéliens est la résultante d'interactions entre facteurs écoclimatiques, socio-économiques et politiques, à l'oeuvre depuis plus de quatre décennies. Au nord du Sénégal, l'espace géographique du Ferlo est représentatif d'une cohabitation entre des systèmes de production agrosylvopastoraux dans laquelle l'activité pastorale se maintient en compétition avec les autres, car les complémentarités anciennes entre systèmes de production agricoles et pastoraux, ou entre pasteurs et collectivité, tendent à se dégrader sous la pression foncière. Ces forces de pression aux intérêts quelques fois divergents ont comme indicateurs de transformation tangibles : la conversion socio-économique et environnementale; la pluralité des modes d'accès à la terre en dehors du cadre légal ; les litiges et difficultés dans l'affectation des terres ; la fragmentation des paysages; la restructuration agricole et pastorale des territoires. C'est aussi dans ce contexte que les politiques de décentralisation et d'aménagement du territoire ont transféré aux collectivités territoriales le pouvoir d'administrer, d'exploiter et de gérer les ressources naturelles et le foncier, afin de renforcer la gouvernance locale et de répondre aux enjeux de développement durable des systèmes de production. L'expérience présentée ici traite du processus de coconstruction et de mise en œuvre d'un outil de simulation pour accompagner et instruire les prises de décision des collectivités locales chargées de l'application de la loi sur le domaine national. Les différentes étapes de cette démarche participative qui ont conduit à la conception, au test et à l'évaluation par les utilisateurs du modèle AIDA et de ses scénarios sont présentées.
\end{abstract}

Mots clés : modélisation ; pastoralisme ; politique foncière ; Sénégal ; système multi-agents.

Thèmes : ressources naturelles et environnement ; productions animales ; territoire, foncier, politique agricole et alimentaire.

\section{Abstract}

An agent-based model to study land allocation policies and their effect on pastoral and territorial dynamics in the Ferlo (Senegal)

The present state of the landscapes and territories in the Sahel is the result of interactions between climatic, economic, social and political factors which have been taking place for more than four decades. In the north of Senegal, the Ferlo geographical space is representative of cohabitation between sylvopastoral and agricultural production systems, within which the pastoral activity is maintained under conflictual conditions as traditional complementarities between agricultural and pastoral production systems, or between pastors and communities, tend to deteriorate under land pressure. Such pressure operates for a variety of often divergent goals displaying tangible transformation indicators with social economic and environmental conversion such as illegal land access plurality, disputes and trouble in land allocation, landscape fragmentation and agricultural territory and pastoral restructuring. It is also within this framework that territory decentralisation and development policies have transferred to stakeholders the power to administer, exploit and manage natural resources and lands under their jurisdiction so as to strengthen local governance and respond to sustainable development needs of production systems. The experiment shown in this case study deals with 
the process of co-building and setting up tools and simulation models in order to accompany and instruct decision making by local communities responsible for applying the law 64-46 of 06 June 1964. The different steps of this participative approach leading to the "AIDA" agent model design and scenarios have been put forward, tested and evaluated by the users.

Key words: land policies; modelling; multi-agent systems; pastoralism; Senegal.

Subjects: animal productions; natural resources and environment; territory, land use, agricultural and food production policy.

$\mathrm{D}$ epuis 1999, les institutions de recherche et d'enseignement supérieur participant au pôle pastoral zones sèches (PPZS) ${ }^{1}$ mènent des activités de recherches interdisciplinaires dans la zone sylvopastorale (ZSP) du Ferlo, au nord-est du Sénégal, pour étudier le fonctionnement des systèmes pastoraux et coconstruire des outils de gestion durable des espaces et des ressources partagés. Les travaux du PPZS partent de l'hypothèse que l'élevage pastoral est la meilleure forme de mise en valeur de ces terres, à condition que l'on préserve la mobilité des troupeaux et que l'on donne aux éleveurs des droits fonciers permettant la sédentarisation partielle des sociétés pastorales, leur diversification et leur sécurisation. Les décisions politiques qui valorisent l'agriculture et la sédentarisation des éleveurs et qui négligent le pastoralisme ont en effet conduit à la surexploitation des parcours et à l'exacerbation des processus de désertification. L'évolution régressive de la végétation observée au Ferlo se traduit par une perte de biodiversité, le vieillissement et la modification de la structure des peuplements ligneux. L'analyse diachronique de l'occupation des sols atteste de la transformation des paysages par la fragmentation et la réduction des parcours (Touré et al., 2003), notamment le long du front agricole du bassin arachidier. L'étude de la mobilité pastorale montre des "territoires de la mobilité " contigus et exigus, liés aux changements des pratiques de mobilité, à la diversification des

\footnotetext{
${ }^{1}$ Le PPZS est une unité de recherche en partenariat qui associe autour de la thématique pastorale, les institutions: suivantes : le Centre de coopération internationale en recherche agronomique pour le développement (Cirad ; France), le Centre de suivi écologique (CSE, Sénégal), I'École nationale d'économie appliquée de Dakar (ENEA; Sénégal), I'Institut sénégalais de recherches agricoles (ISRA), I'Université Cheikh Anta Diop (Ucad) de Dakar.
}

pratiques d'exhaure, de transport et de gestion de l'eau (Diop et al., 2003) et au recours au salariat. La mutation des systèmes sociaux se traduit aussi par la diversification des activités, l'apparition de flux externes, l'émergence d'une diversité des propriétaires de troupeaux et la restriction des accès aux ressources (Ancey et al., 2003).

La finalité centrale de notre modélisation est de savoir comment représenter les pratiques pastorales et leurs évolutions en interaction avec les dynamiques spatiales pour accompagner l'aménagement territorial durable du Ferlo.

Trois questions secondaires en découlent : - comment concevoir et socialiser les outils et méthodes de modélisation?

- quels indicateurs partagés (spatiaux, socio-économiques, écologiques) choisir pour représenter les dynamiques des systèmes pastoraux?

- comment coconstruire une modélisation prospective?

\section{Le Ferlo: un espace pastoral en transition}

Doté de potentialités fourragères naturelles, le Ferlo est emblématique d'un pastoralisme séculaire traversé par des changements de pratiques, des flux commerciaux, des enjeux fonciers contrastés du sud au nord et sur ses marges. Jadis, le mode de transhumance s'opérait entre la vallée du fleuve Sénégal Waalo où les animaux résidaient en saison sèche, et le Jeeri, traversé par la vallée du Ferlo, aux alentours des puits pastoraux, en saison des pluies (Barral et al., 1983). La politique d'hydraulique pastorale lancée par l'administration coloniale pour améliorer la ressource en eau et préserver le caractère pastoral du Ferlo a restructuré profondément l'espace et les pratiques pastorales, en substituant à l'agencement des différents pâturages soumis à des droits prioritaires des éleveurs (" burum ") une vaste zone régulièrement maillée de forages (Touré et al., 1997). Dans les années 1970, le développement de l'agriculture irriguée dans la vallée du fleuve Sénégal et autour du lac de Guiers, ainsi que l'expansion du bassin arachidier à l'ouest et au sud ont contribué à la réduction de l'espace pastoral du Ferlo (Touré et al., 2004).

La loi 64-46 du 17 juin 1964 a créé un vaste domaine national, puis les décrets fonciers de 1972 et de 1987 ont rendu effective la gestion foncière des terres par les communautés rurales, mais ils ont privilégié la mise en valeur agricole aux dépens des activités pastorales (Caverivière et Debene, 1988). Dans le contexte de la réforme du foncier en cours, l'enjeu est d'aider les élus locaux à décrypter les intérêts et stratégies des acteurs pour procéder à des arbitrages plus cohérents dans le processus d'affectation des terres. Apriori, le pastoralisme n'est en effet pas suffisamment reconnu dans ce processus, puisque la notion même de mise en valeur des terres, qui prime dans toute décision d'affectation, privilégie l'habitat sédentaire et la culture fixée. Cependant, dans les communautés rurales à forte composante pastorale, les conseils ruraux sont composés en grande partie d'éleveurs et les pasteurs ont pu négocier des accords durables d'usage des terres et d'accès à l'eau.

Notre site d'étude, compris entre le lac de Guiers et Tatki, est bordé par la vallée du fleuve Sénégal au nord et le système des vallées fossiles au sud. Il est situé entre les régions administratives de Saint-Louis et Louga, et s'étend sur une vingtaine de collectivités territoriales. Cet espace pastoral est structuré à l'est par le maillage des forages qui polarisent autour de leur aire de desserte des campements de saison des pluies et de saison sèche (figure 1). L'alternance de dunes à substrat limono- 


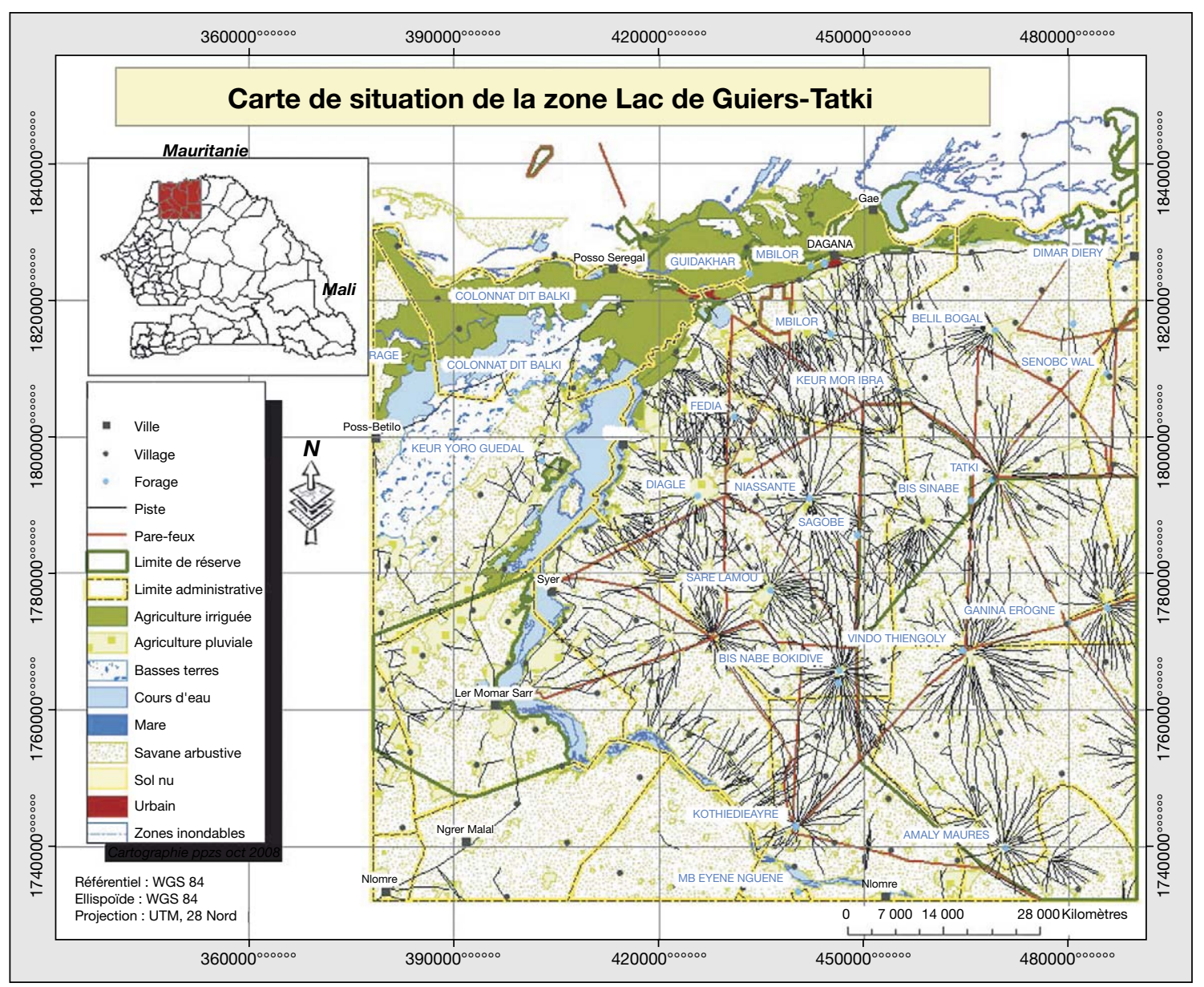

Figure 1. Localisation du site d'étude Lac de Guiers-Tatki.

Figure 1. The study area: Guiers-Tatki Lake.

sableux et de couloirs interdunaires sabloargileux jalonnés de mares détermine les unités physiographiques comportant une végétation essentiellement arbustive très éparse, constituée de Boscia senegalensis, Balanites aegyptiaca et Calotropis procera. La strate herbacée est composée d'espèces annuelles formant un tapis vert continu en saison humide de Aristida mutabilis, Schoenefeldia gracilis, Chloris prieurii et Eragrostis tremula. Une saison sèche - huit à neuf mois - et une saison des pluies - trois à quatre mois - rythment le fonctionnement des écosystèmes et les activités socio-économiques dans ce milieu.

Le développement de l'agriculture irriguée exerce une pression accrue, rendant difficile l'accès du bétail aux pâturages. À cela, s'ajoute la diversité, au niveau local, des intérêts des notables (politiques et professionnels), et des cadres de concertation ou de négociation qui placent les représentants de l'État et les élus locaux dans un flou, voire un vide juridique (Diop Gaye et al., 2007). À côté du droit moderne, seule voie légale d'accès à la terre, il existe une grande variété de modes d'accès légitimes à la terre, du point de vue de la coutume, parmi lesquels le don, le prêt, l'héritage, l'achat et la location (Ndiaye, 2005). De nombreux litiges et conflits fonciers relevés sont souvent soumis au conseil rural (CR) : multi-affectations d'une même parcelle; affectations à des personnes non identifiées; difficultés à désaffecter; inexistence d'un cadastre rural. Le CR est en effet l'organe délibérant de la communauté rurale depuis 1972, donc le seul habilité à gérer les terres des "zones de terroir ". Toutefois, il reste limité dans son action par la faiblesse de ses moyens financiers et techniques. Dès lors, la tendance à la dégradation des terres et la compétition foncière, suite à l'arrivée progressive de grands exploitants nationaux et étran- gers, compliquent davantage l'accès au foncier des petits exploitants.

\section{Quels enjeux} et positionnement pour une rechercheaction?

La définition des objectifs de la modélisation est un exercice périlleux (Fourage et al., 2006). Elle pose la question du positionnement éthique et scientifique des chercheurs. Ils se doivent de clarifier leurs intentions, lesquelles sont très souvent fondées sur une éthique du développement raisonné, parfois sur une idéologie, voire sur une éthique de l'engagement. Ils ne sont les mandataires d'aucune catégorie d'acteurs et devront donc se garder soigneusement de tout énoncé en terme de 
valeur (légal/illégal, spoliation, accaparement, favoritisme, spéculation...) et se concentrer sur une analyse approfondie de la situation sociale. Les objectifs de la simulation informatique ne sont pas d'instaurer un changement dans les pratiques des acteurs, ce qui risquerait d'être perçu comme le fruit d'un interventionnisme prescriptif exogène. Il s'agit de créer les conditions de la concertation entre acteurs autour de l'affectation des terres, laquelle est un objet sensible et constitue un enjeu socio-économique majeur : toute modification dans les règles qui la définissent est porteuse d'une remise en cause des autorités et de l'ordre social régulé. La simulation est donc considérée ici comme un outil de médiation. Ce sont les discussions entre utilisateurs qui pourront, le cas échéant impulser des changements dans les pratiques.

L'affectation et la désaffectation des terres cristallisent les enjeux du développement durable de l'agriculture et de l'élevage du site d'étude. Elles suscitent espoirs et controverses et sont l'objet de projections et représentations contradictoires. Nous avons choisi de modéliser ces enjeux à partir des travaux des ateliers de conception participative initiés avec des représentants (réunis dans un comité d'utilisateurs) des communautés villageoises, qui bordent le lac de Guiers et les aires de desserte des forages pastoraux.

\section{De la coconstruction de modèles de simulation à la prospective}

\section{Démarche}

La coconstruction du "modèle multiagents" AIDA (Analyse des impacts des décisions d'affectation) pour l'étude des interactions entre élevage et territoire s'appuie sur la méthode AGRRO (Agent, groupe, rôle, ressource, objet) formalisée par Bah (2008). Les enjeux de la démarche sont de s'assurer de l'utilité, de l'" utilisabilité " et de l'acceptabilité du modèle en proposant un cadre de travail intégrant : a) les phases de la conception ; b) des mécanismes de régulation (de la prise de tour de parole, des droits intellectuels, etc. ; c) une base d'expériences antérieures ; d) un support d'échange de connaissances structurées ; e) un cadre matériel de communication et de travail (Caelan, 1996). AGRRO est un processus itératif, incrémental et souple. En effet il se décompose en plusieurs ateliers, eux-mêmes structurés en plusieurs phases étalées dans le temps (figure 2), avec un produit qui évolue de manière incrémentale entre chaque atelier, selon une approche adaptative qui tient compte des réactions des acteurs.

Sur la base d'un diagnostic participatif qui a fait ressortir une forte demande pour un outil de gouvernance foncière au niveau du conseil rural, nous avons procédé à la phase de conception participative de l'outil AIDA (Bah et al., 2008). Celle-ci a débuté par un atelier, combinant jeux de rôles et travaux de groupe (Bah et al., 2006), qui a exploré les enjeux liés à la procédure d'affectation des terres, et s'est poursuivie par un atelier de validation du module d'initialisation interactif de l'outil.

\section{Modèle conceptuel de AIDA}

Dans le contexte du foncier et des interactions entre pastoralisme et territoire dans la zone étudiée, notre modèle multi-agents est composé de différentes ressources :

$R=\{$ ressource eau, ressource ligneuse, ressource berbacée $\}$,

d'objets :

$$
\begin{gathered}
0=\{\text { objet pare }- \text { feu, objet aménagement }, \\
\text { objet infrastructure... }\}
\end{gathered}
$$

et d'acteurs :

$A=\{$ acteur local, acteur étranger, acteur collectif $\}$

pouvant jouer plusieurs rôles :

$$
R_{0}=\{\text { rôle agriculteur, rôle éleveur... }\}
$$

dans un espace foncier découpé en parcelles (P) (figure 3). Chaque acteur a ${ }_{i} \in A$ joue un ou plusieurs rôles

$$
\left\{r_{\text {oj }} \ldots\right\} \in R_{0} \text { avec } R_{O}=R_{o}^{1} \cup R_{O}^{2} \cup R_{o}^{3}
$$

$R_{0}^{1}$ représente les rôles de production et économiques au sens large (rôles professionnels) :

$$
\left.\begin{array}{r}
R_{0}^{1}=\{\text { rôle agriculteur, } \\
\text { rôle éleveur, rôle pêcheur, } \\
\text { rôle commerçant ... }
\end{array}\right\}
$$

$R_{0}^{2}$ représente les rôles politiques, électifs et techniques. Ce sont les gestionnaires du foncier :

$R_{o}^{2}=\left\{\begin{array}{l}\text { rôle conseiller rural, }, \text { rôle president communauté rurale } \\ \text { rôle commission domaniale, rôle sous préfet, ... }\end{array}\right\}$,

$R_{0}^{3}$ représente les rôles religieux et sociaux qui peuvent parfois influer sur les décisions d'affectation :

$$
R_{0}^{3}=\{\text { rôle chef coutumier, rôle marabout }, . . .\}
$$

Il est possible à travers la fonction rôle de spécifier le ou les rôles de chaque acteur:

$$
\text { rôle }: a_{i} \rightarrow\left\{r_{o j, \ldots}\right\} \in R_{o}, a_{i} A
$$

Nous nous intéressons particulièrement dans ce modèle aux rôles $r_{0 j}=$ "rôle éleveur" et rok = "rôle agriculteur" dans leurs rapports avec la dynamique territoriale. L'acteur dans son rôle d'éleveur exprime une demande affectation en fonction de ses besoins en terme de foncier. Si la demande est acceptée par le conseil rural et validée par le sous-préfet, l'acteur est installé (figure 4). Il doit, pour abreuver son troupeau, se mettre en quête de points d'eau et privilégier les parcours qui lui fourniront un pâturage de qualité (figure 5) en quantité suffisante. Cette

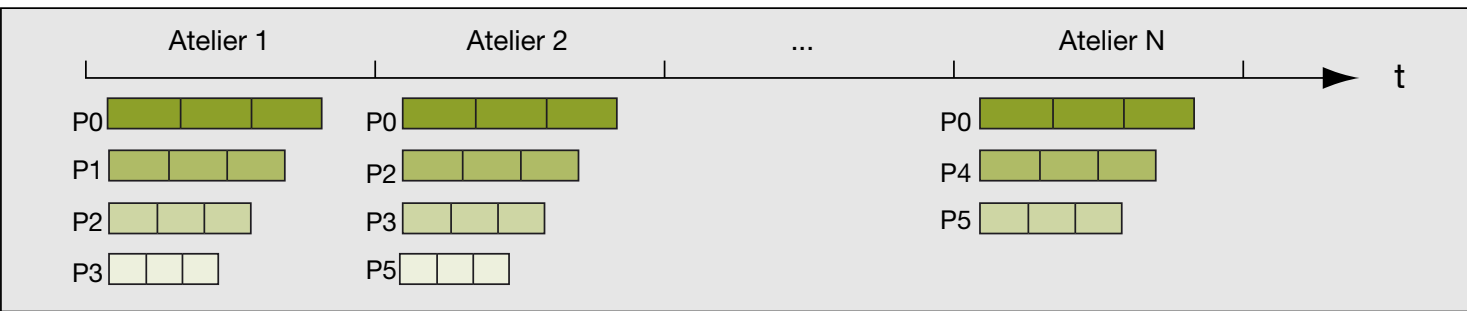

Figure 2. La démarche AGRRO (Agent, groupe, rôle, ressource, objet).

Figure 2. AGRRO Approach.

P0 : planification ; P1 : analyse participative ; P2 : conception participative ; P3 : implémentation ; P4 : restitution et tests ; P5 : déploiement et appropriation. 


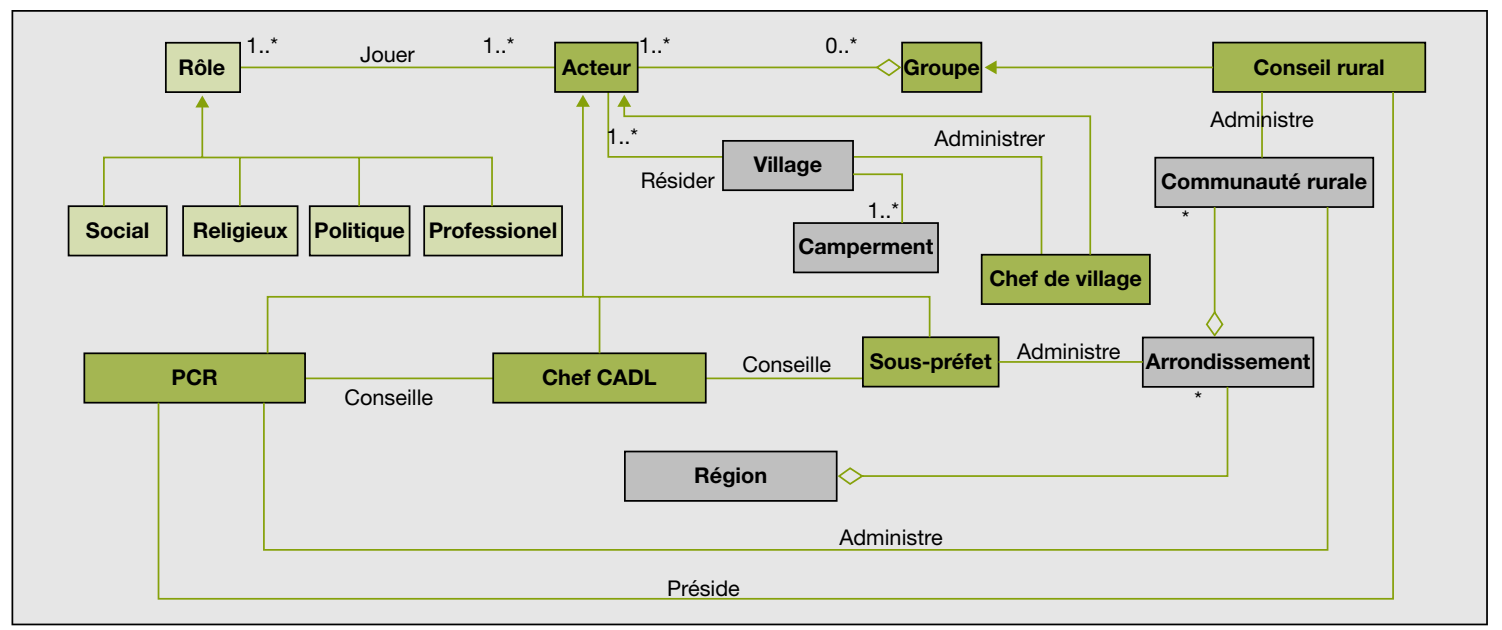

Figure 3. Diagramme de classes des acteurs et de leurs rôles.

Figure 3. Class Diagram of actors and their roles.

PCR : président de communauté rurale; Chef CADL : chef du centre d'appui au développement local).

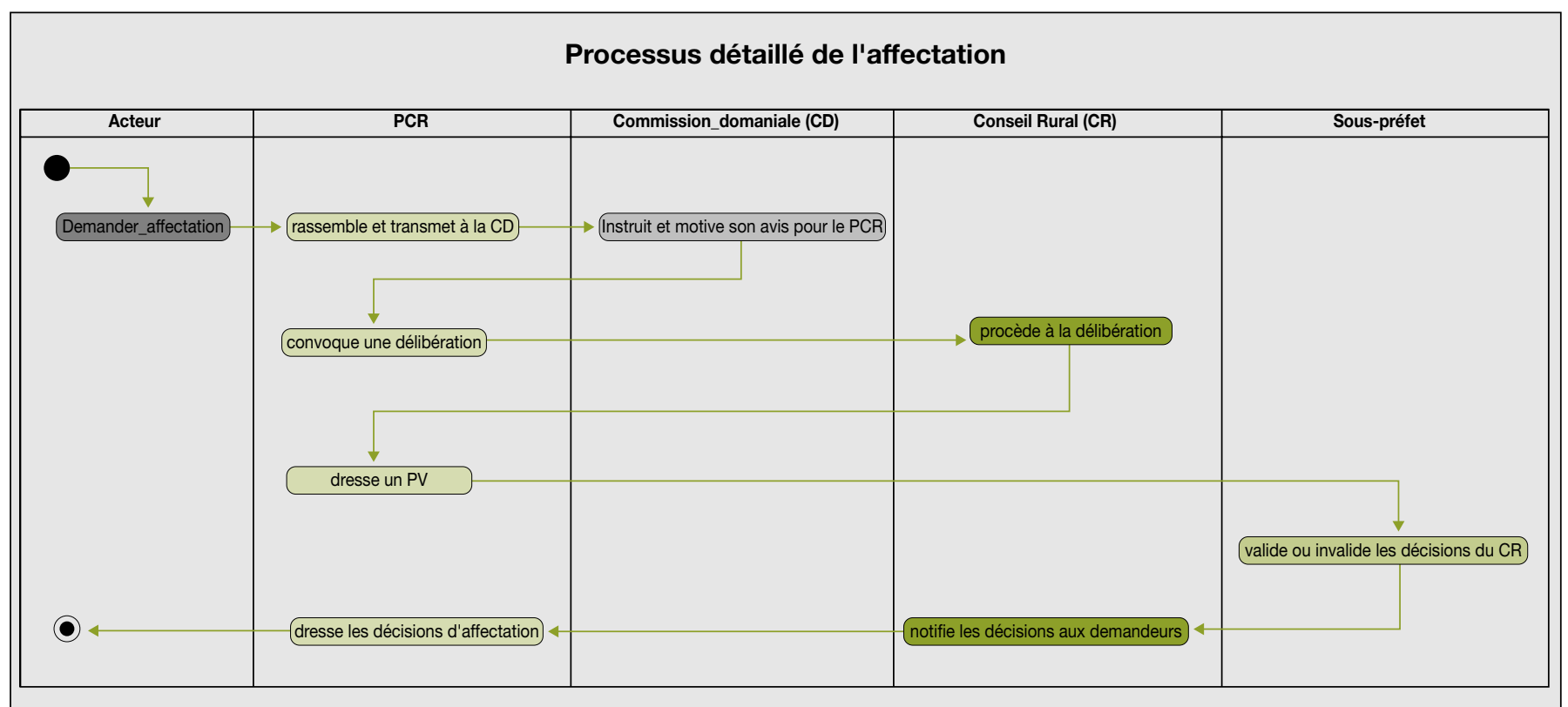

Figure 4. Processus d'affectation des terres et interaction entre acteurs selon les rôles joués.

Figure 4. Land allocation process, roles interactions.

PCR : président de communauté rurale ; PV : procès-verbal.

quête a un coût qui dépend de la distance parcourue et de la configuration spatiotemporelle des ressources pastorales.

L'acteur dans son rôle d'agriculteur, privilégiera les parcelles irriguées ou ayant un accès à l'eau, de bonne qualité, éloignées des terres de parcours, faciles d'accès, etc. (figure 5). Exploiter la parcelle a aussi un coût, qui dépend des intrants utilisés et de la main-d'œuvre disponible.

\section{Description de l'outil développé: AIDA}

AIDA consiste en deux modules interdépendants :

1) un module d'initialisation interactif, qui permet au conseil rural de renseigner et placer sur une carte des affectations (demandées et attribuées) et ainsi d'alimenter une base de données foncière ;
2) un module de simulation pour venir en appui à l'évaluation des impacts des décisions d'affectation, implémenté dans l'environnement de développement CORMAS (Bousquet et al., 1998). Le simulateur intègre plusieurs couches spatiales. La figure 5 montre l'interface principale de simulation représentant la carte d'aptitude des sols autour du lac de Guiers. Du point de vue de son fonction- 


\section{Début}

\section{Encadré 1}

1) Prise en compte de la qualité de la saison des pluies.

2) Les acteurs effectuent leurs demandes de parcelles en fonction de leurs besoins (figure 4).

3) Le conseil rural annonce au besoin une date et procède à la délibération (figure 4).

4) Les acteurs exploitent leurs parcelles ou s'occupent de leurs troupeaux (figure 6).

Fin

nement, à chaque pas de temps (le mois), sont effectuées des séquences d'actions (encadré 1).

Dans le modèle, l'évolution de la ressource pastorale est fonction de la pluviométrie. Elle suit la loi de Boudet (Boudet, 1987) :

$$
B h=4,1 P-515
$$

où Bh est la biomasse herbacée produite en $\mathrm{kg}$ de matière sèche/hectare et $\mathrm{P}$ la pluviosité annuelle en $\mathrm{mm}$.

\section{Des scénarios pour débattre du développement durable de l'élevage en zone sahélienne}

Nous avons développé plusieurs scenarii autour de la pluviométrie, des politiques d'affectation et des types d'exploitation à l'échelle des communautés rurales. Pour ces simulations, nous avons retenu cinq campements d'éleveurs et cinq campements d'agriculteurs par communauté rurale. La population d'éleveurs est en début de simulation au nombre de 10 par campement, la taille des troupeaux est de 30 unités de bétail tropical (UBT) en moyenne et le nombre d'affectations évolue dans le temps en fonction de la pluviosité. Le pas de temps est le mois. Nous avons ensuite construit les simulations, sur 30 ans, en croisant plusieurs scenarii et en réalisant des études de sensibilité sur les différents paramètres du modèle. Nous exposons ici les résultats de deux des huit scénarios développés :

- scénario 2: l'agriculture irriguée et l'agro-industrie sont privilégiées et la pluviométrie est à tendance moyenne à bonne ;

- scénario 3: l'agriculture irriguée et l'agro-industrie sont privilégiées et la plu-

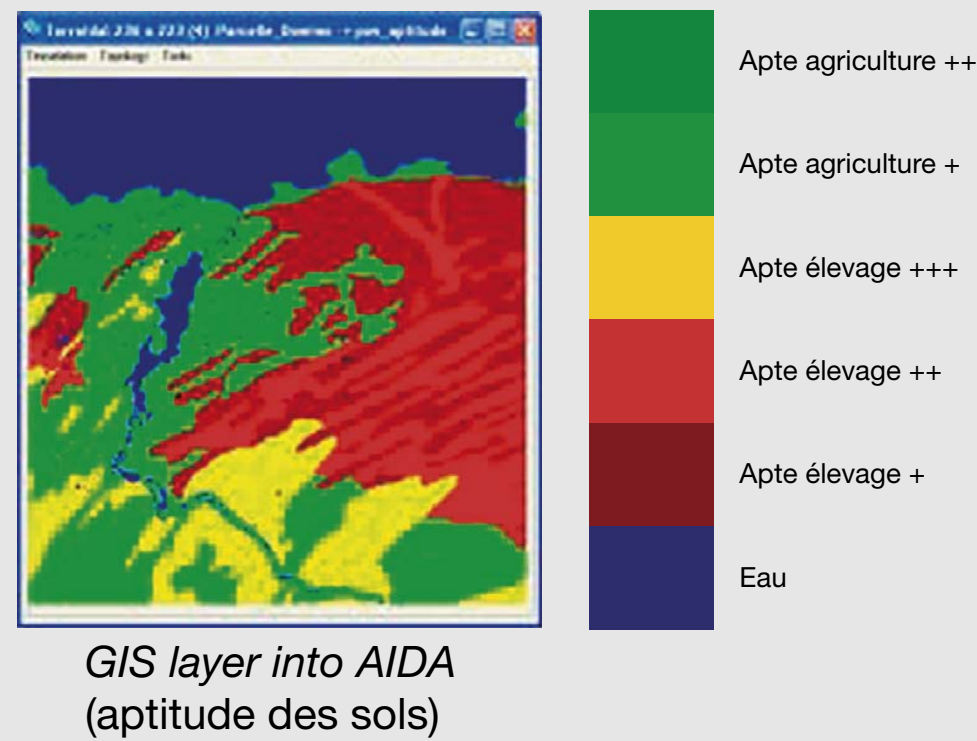

Figure 5. Espace de simulation (couche d'information).

Figure 5. Simulation Space, information layer.

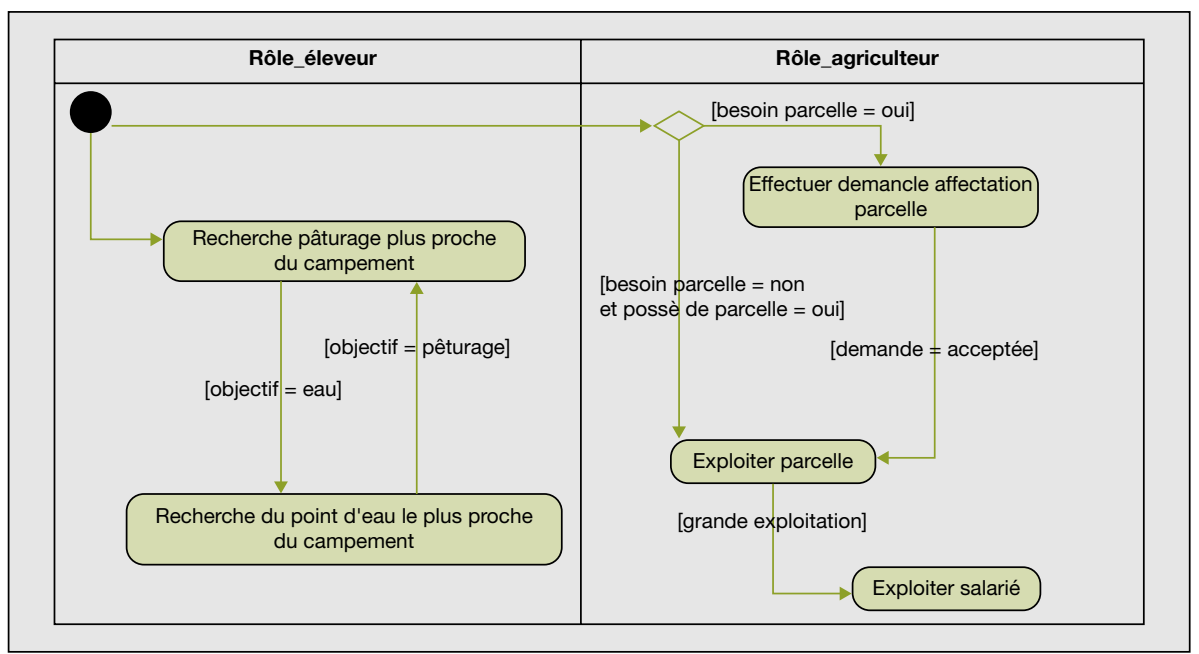

Figure 6. Explicitation des principaux rôles développés dans le simulateur AIDA.

Figure 6. Main roles developed in the AIDA simulator.

viométrie est à tendance moyenne à mauvaise.

Pour chaque simulation nous proposons plusieurs indicateurs (ou sorties) :

- l'indice de Clarck-Evans $-2 \bar{r} \sqrt{\lambda}$, où $\bar{r}$ est la moyenne des distances au plus proche voisin et $\lambda$ la densité globale ou nombre d'affectations par unité de volume de l'espace des affectations -, pour étudier la distribution spatiale des affectations. Il mesure le degré d'agrégation des affectations. S'il est supérieur ou égal à 1 on est dans le cas d'une distribution au hasard, s'il est inférieur à 1 nous avons une tendance au regroupement des affectations (Treuil et al., 2008) ;

- le nombre de champs traversés par le bétail, source de conflits potentiels entre agriculteurs et éleveurs durant la campagne agricole ;

- l'évolution du nombre d'éleveurs dans la zone d'étude ;

- l'évolution du nombre d'affectations de terre. 


\section{Premiers résultats : tests et validation du modèle}

Les premiers résultats permettent de tester et de "valider" la pertinence de nos scénarios et indicateurs pour la compréhension des interactions élevage et dynamiques territoriales. Ce travail exploratoire montre, à travers les deux scénarios, une diminution notable du nombre d'éleveurs (figure 7), une augmentation significative des affectations destinées aux projets agricoles (figure 8) et une fragmentation de l'espace (figure 9). Ces résultats de simulation, confirmés par nos enquêtes de terrain (Daré et al., 2007), mettent en exergue une pression accrue sur le foncier pastoral rendant difficile l'accès ancestral du bétail aux pâturages et, de fait, une marginalisation du pastoralisme par l'exclusion de la population des pasteurs, des infrastructures scolaires, administratives, ou de santé, parce qu'elle est repoussée loin des villages.

De plus, il a été constaté que les rejets de l'industrie agroalimentaire et de l'agriculture - les scénarios 2 et 3 favorisent l'agroindustrie - menacent la qualité des eaux du lac, avec la prolifération de Typha autralis rendant difficile l'abreuvement du bétail et avec le développement de parasites responsables de la douve du foie affectant particulièrement les ovins. Il existe donc un risque sanitaire réel par défaut d'entretien et pollution des rives du lac de Guiers, qui concerne hommes et animaux

Par ailleurs, la forte valeur de l'indice Clarck-Evans (figure 9) corrobore l'absence de planification des affectations dans certaines communautés rurales. Cela contribue à la fragmentation de la trame foncière. Cette configuration spatiale émiettée est à l'origine de conflits entre agriculteurs et éleveurs lors des campagnes agricoles (nombre de champs traversés élevés) (figure 10), d'où l'intérêt (pas encore démontré) pour les communautés rurales de mettre en place des plans locaux de développement et des plans d'occupation et d'affectation des sols.

\section{Conclusion}

Les ateliers participatifs pour la maîtrise du foncier et des interactions élevage et territoire menés au niveau du lac de Guiers-

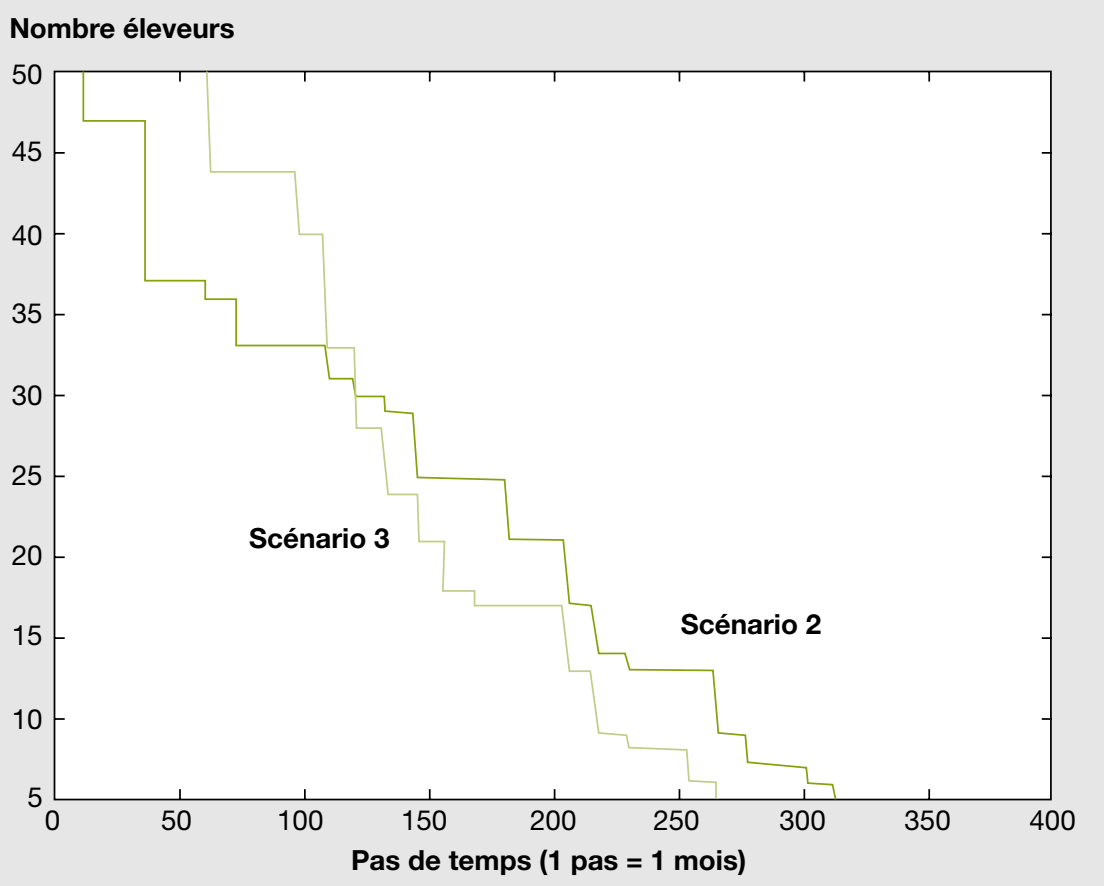

Figure 7. Évolution du nombre d'éleveurs.

Figure 7. Breeder dynamics.

Affectations (hectares)

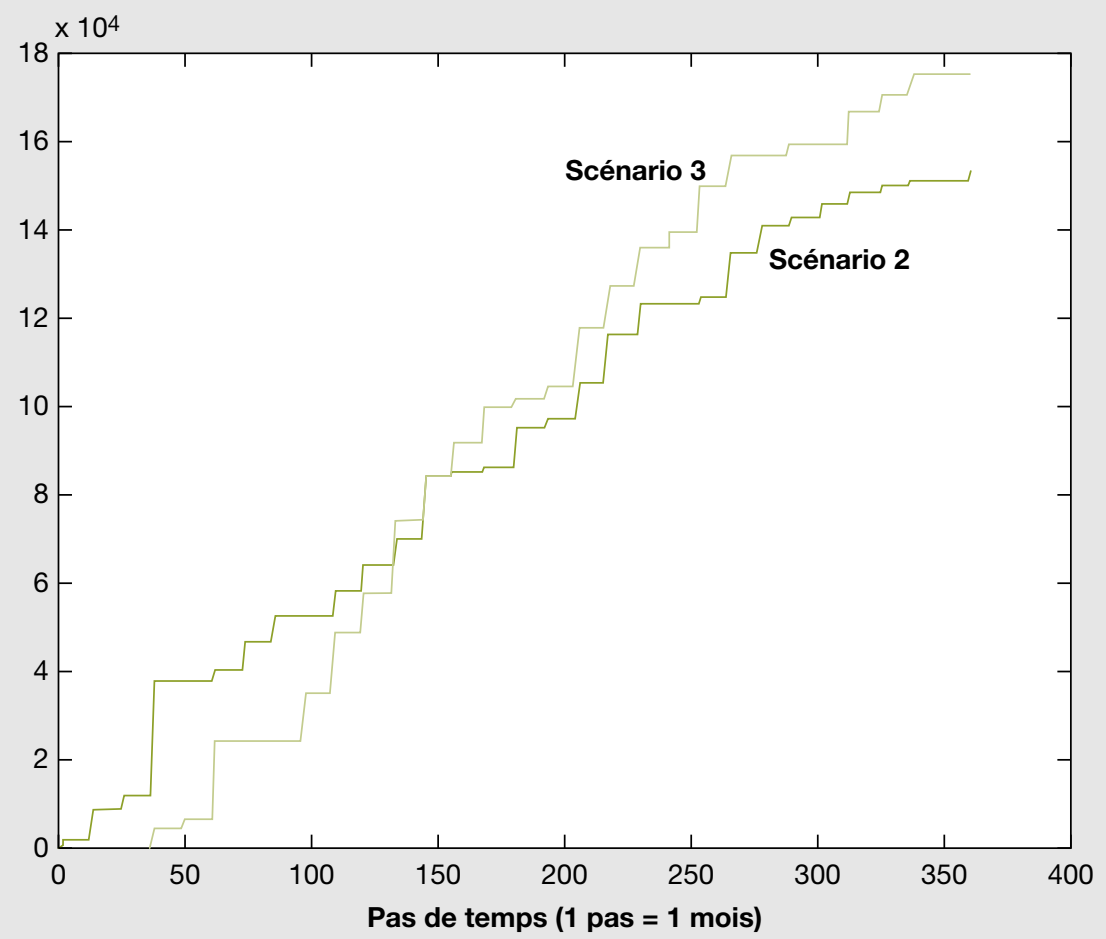

Figure 8. Évolution des affectations de terres.

Figure 8. Land allocation dynamics. 


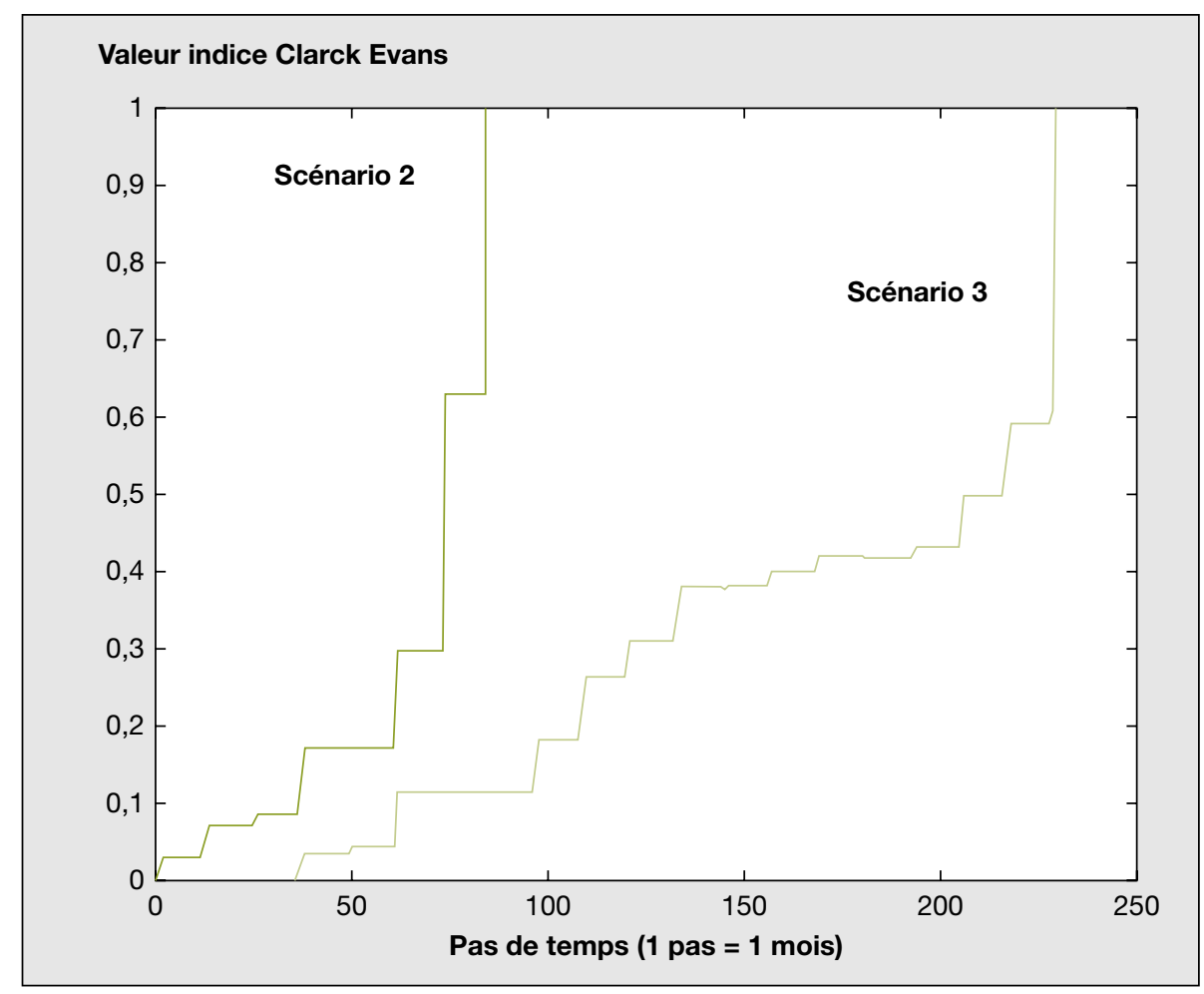

Figure 9. Évolution de l'indice Clarck-Evans.

Figure 9. Evolution of the Clark-Evans index.

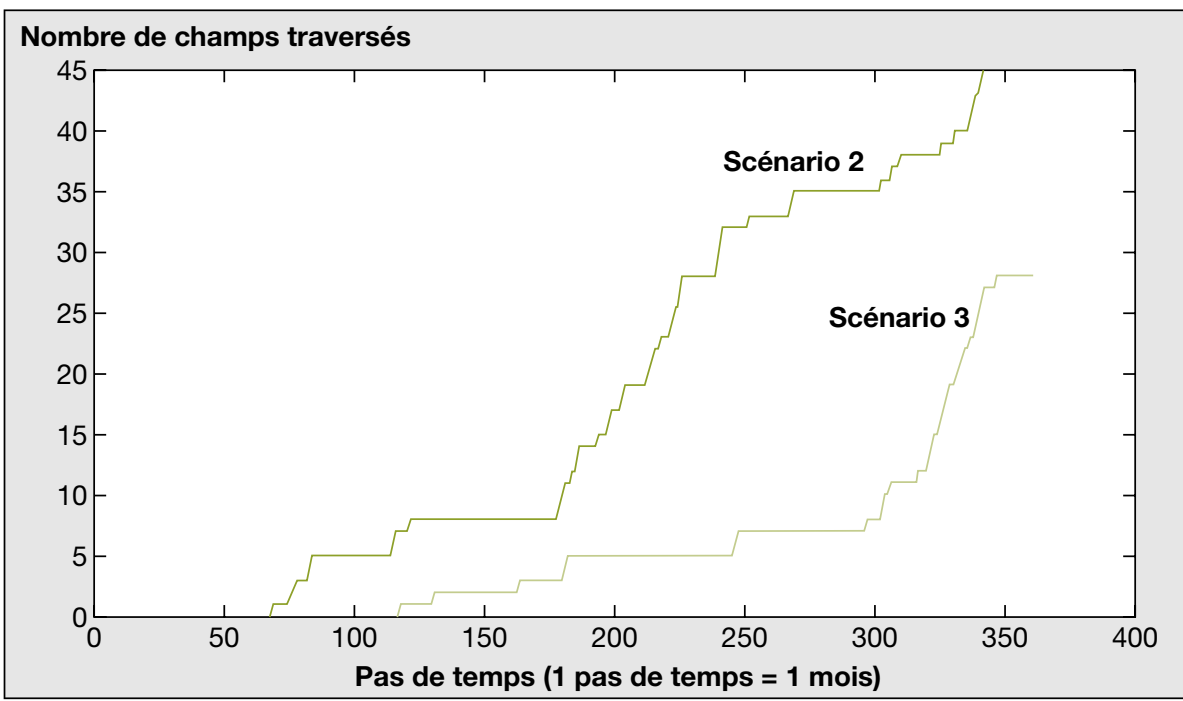

Figure 10. Nombre de champs traversés.

Figure 10. Number of crossed fields.

Tatki ont permis de développer différents outils d'accompagnement pour les acteurs : cartes à dire d'acteurs; bases de données; modèle de simulation agent. L'analyse des logiques sociales relatives aux usages agricoles et à la dynamique montrent la pertinence des indicateurs tirés de la confrontation de différents types de connaissances (scientifique, pratique, technique). Ainsi, au moyen du degré d'agrégation des affectations, de l'estimation du nombre de champs traversés par le bétail et du nombre d'affectations pour une période donnée, nous sommes en mesure de fournir une représentation territoriale qui pourra être partagée par l'ensemble des acteurs et leur permettra de se projeter dans l'avenir de leur terroir.

La justification sociale du processus exposé dans ce travail est à trouver du côté des usagers (éleveurs et agriculteurs) et des décideurs (président de communauté rurale, sous-préfet...): soit la démarche fait sens et une dynamique de gestion concertée et raisonnée s'enclenche, soit elle est vécue comme étrangère aux préoccupations des acteurs et les conditions de la régulation de l'affectation des terres peineront à se dessiner. Toutefois, la démarche suivie dans notre étude de cas vient compléter et enrichir l'expérience réussie des plans d'occupation et d'affectation des sols (D'Aquino et al., 2001) développée avec les collectivités territoriales du delta du fleuve Sénégal. Par ailleurs, le retour d'expérience montre une appropriation certaine des ces différents outils de planification et de gestion des ressources naturelles par les collectivités locales dans la mise en ouvre du processus de décentralisation.

\section{Références}

Ancey V, Diao A. The vulnerability of the pastoral systems in arid and semi- arid areas: What are the relevant indicators of the risks in the Sahel? In: Allsopp N, ed. Proceeding of the VII International Rangelands Congress Editions. Durban : SB Conference, 2003.

Bah A. Conception Participative de Simulations Multi-agents : Cadre Méthodologique. Thèse de doctorat : spécialité Informatique, ED SEIB, École pratique des Hautes Études, ParisSorbonne, 2008

Bah A, Touré I, Le Page $C$, et al. An agentbased model to understand the multiple uses of land and resources around drillings in sahel. Mathematical and computer modelling 2006; $44: 513-34$.

Barral H. Systèmes de production d'élevage au Sénégal dans la région du Ferlo. Dakar: Orstom-Isra, 1983.

Boudet G. Connaissance et gestion de l'espace pastoral sahélien. In: Audru J, Boudet G, Cesar J, et al., eds. Terroirs pastoraux et agropastoraux en zone tropicale. Gestion, aménagement et intensification fourragère. Études et synthèses, numéro 24. [. Cirad-IEMVT, 1987 [ville d'édition]. 
Bousquet F, Bakam I, Lepage C. et al. Cormas: Common-Pool Resources and Multi-agent Sys tems. Actes de la $11^{\mathrm{e}}$ Conference Internationale sur les applications industrielles et $d^{\prime}$ ingénierie de I'Intelligence Artificielle et des Systèmes Experts, Castellon (Espagne). Berlin, Springer-Verlag, 1998.

Caelan J, et al. Nouvelles interfaces hommemachine. Paris : OFTA éditions, 1996.

Caverivière, M., M. Debene. Le droit foncier sénégalais. Mondes en devenir. Paris : BergerLevrault, 1988.

D'Aquino P, Seck SM. Et si les approches participatives étaient inadaptées à la gestion décentralisée de territoire? Géocarrefour $2001 ; 76: 233-9$
Daré W, Fourage C, Gaye ID. Positionnement des sociologues dans la démarche de modélisation Domino. NPSS $2007 ; 2$ : 103-26.

Diop AT, Sy O, Ickowicz A, et al. Transhumance and nomadism in the sylvopastoral region (Ferlo) of Senegal: perspectives for pastoral development. In : Allsopp N, ed. Proceeding of the VII International Rangelands Congress. Durban: SB Conference, 2003.

Fourage C, Daré W, Diop Gl, et al. Methodological issues arisen from the involment of sociologistes in a companion modelling approach : interests and limits. Actes du $14^{\mathrm{e}}$ congrès mondial de sociologie de I'ISA, Durban, 2006.
Touré I, Bah A, D'Aquino P, et al. Savoirs experts et savoirs locaux pour la coélaboration $\mathrm{d}^{\prime}$ outils cartographiques d'aide à la décision Cah Agric 2004 ; 13 : 546-53.

Touré I, Diop AT, Diouf A, et al. Dynamic analysis of landscapes and landcovers for the knowledge and evolution of the pastoral ecosystems in the Ferlo-Senegal Proceeding of the VII International Rangelands Congress Editions. Durban: SB Conference, 2003.

Touré O. La gestion des ressources naturelles en milieu pastoral, L'exemple du Ferlo sénégalais. Tome 1. Dakar, Paris: Société-Espace-Temps; Karthala, 1997.

Treuil JP, Drogoul A, Zucker JD. Modélisation et Simulation à base d'agents, Exemples commentés, outils informatiques et questions théoriques. Paris: Dunod, 2008. 